\title{
Aquaculture Drugs: Sources, Active Ingredients, Pharmaceutic Preparations and Methods of Administration
}

Vikash Kumar* and Suvra Roy

Central Inland Fisheries Research Institute (CIFRI), Barrackpore, India

\begin{abstract}
Aquaculture remains a growing, vibrant and important food production sector. However, disease outbreaks have disrupted aquaculture production, often with severe socio-economic and ecological consequences. Anti-microbial chemotherapy has been used in aquaculture for over past 60 years and discovery of new and effective anti-microbial agents have changed the microbial virulence and severity of disease, leading to a dramatic reduction in morbidity and mortality, and contributing to significant advances in the health of the general population. Anti-microbials are used both as prophylactic, at times of heightened risk of disease and as therapeutic, when an outbreak of disease occurs in the system. There are several anti-microbials drugs used for fish health management however, information on anti-microbial used in aquaculture and pharmacokinetics and pharmacodynamics of administration is very limited. In recent years, the issues relating to antibacterial use in animal food is under severe scientific and public scrutiny due to human health concern. Besides providing protection against microbial infection, chemotherapy may complicate health management by triggering toxicity, resistance, residues and occasionally public health and environmental consequences. Therefore, highly efficient and relatively safer drug is required to develop with the emphasis on prevention, which is likely to be more cost-effective than cure, which will reduce the possible problems associated with excessive use anti-microbials.
\end{abstract}

Keywords: Aquaculture; Antimicrobial chemotherapy; Aquaculture drugs; Health management

\section{Introduction}

Aquaculture is one of the fastest-growing food-producing sector worldwide, increasing at an average rate of $7.1 \%$ per year, and contributes $46 \%$ of total food fish supply [1]. However, disease outbreak is considered to be a significant constraint to the development of this sector, causing huge economic losses per year worldwide [2,3]. Consequently, a wide range of anti-microbial, including antibacterials (antibiotics), pesticides, hormones, anaesthetics, pigments, minerals and vitamins, are used to avoid or control diseases in aquaculture (Table 1) [4-7]. Apart from broodstock facilities anti-microbial are also frequently used in hatcheries to prevent the introduction of pathogens to new facilities when eggs, fry, or broodstock are moved; to prevent the spread of disease to wild fish via the hatchery effluent or when hatchery fish are released or stocked out; and to prevent the amplification of pathogens already endemic in a watershed [8-10]. Moreover, information on antimicrobial used in aquaculture and pharmacokinetics (PK) and pharmacodynamics (PD) of administration is very limited for most of the farmed species [11-14].

Anti-microbial (natural or synthetic origin) that are sufficiently non-toxic to the host are generally used as chemotherapeutic agents for the treatment of infectious diseases. The choice of anti-microbial for the treatment of diseases are becoming increasingly limited and expensive and, in some cases, unavailable due to the emergence of drug resistance in bacteria that is threatening to reverse much of the medical progress of the past 60 years [15]. In aquaculture, anti-microbial have been mainly used for therapeutic purposes and as prophylactic agents [15-17]. The use of anti-microbial as growth promoters in any aspect of aquaculture is generally rare. As prophylactic agents, they are used mostly in confined hatchery, the juvenile or larval stages of aquatic animal production. Despite the widespread use of anti-microbial in aquaculture facilities, limited data is available on the specific types and amounts required to control infection and disease and till date there are no specific anti-microbial agents has been developed for aquaculture use $[17,18]$. The anti-microbial used to treat aquatic animals will be different that are cultured in net pens or cages as compared to those

\begin{tabular}{|c|c|}
\hline \multirow{4}{*}{ Disinfectants } & Formaldehyde \\
\hline & Potassium permanganate \\
\hline & Copper sulfate \\
\hline & Chloramine-T \\
\hline \multirow{3}{*}{ Pesticides } & Diflubenzuron \\
\hline & Malathion \\
\hline & Methyl parathion \\
\hline \multirow{6}{*}{ Antibiotics } & Florfenicol \\
\hline & Oxytetracycline \\
\hline & Enrofloxacin \\
\hline & Chloramphenicol \\
\hline & Erythromycin \\
\hline & Trimethoprim + Sulfametoxazol \\
\hline
\end{tabular}

Table 1: Frequently used substances in the treatment of fish diseases.

held in an indoor facility or an aquarium. The treatment process must be feasible and an appropriate treatment route for fish, e.g., aquarium or selected broodstock individuals must be used commercial aquaculture ventures. The stress associated with treatments must be balanced with the need for and the expected benefits of treatment $[7,14]$. Approved pharmaceutical agents (by FDA) available for aquaculture usage are safe and effective and follow the rules and regulations i.e., minimum or no unexpected side effects (adverse effects) should be associated with the drug, product's active ingredients come from reliable sources, and follows good manufacturing practices while making the product and the

*Corresponding author: Vikash Kumar, Central Inland Fisheries Research Institute (CIFRI), Barrackpore, 700120, India, Tel: 033-25920177; E-mail: kumar.vika.vikash2@gmail.com

Received July 24, 2017; Accepted October 27, 2017; Published October 30, 2017

Citation: Kumar V, Roy S (2017) Aquaculture Drugs: Sources, Active Ingredients, Pharmaceutic Preparations and Methods of Administration. J Aquac Res Development 8: 510. doi: 10.4172/2155-9546.1000510

Copyright: ( 2017 Kumar V, et al. This is an open-access article distributed under the terms of the Creative Commons Attribution License, which permits unrestricted use, distribution, and reproduction in any medium, provided the original author and source are credited. 
drug product to be used in a food-producing animal, residues in food products (such as meat and milk) from that animal must be shown to be safe for human consumption (Table 2). Some reviews have already documented the drugs used in aquaculture $[19,20]$ and they mainly focused on their effects in the aquaculture species and their harmful impacts. The present review focuses on the importance of aquaculture drugs, its source, active ingredient, pharmaceutic preparation and method of administration.

\section{Sources of Drugs}

Drug may be obtained from many different sources. Many foods may serve under some conditions as both source of nutrition and of medication simultaneously. Natural products (NPs) play an important role in drug discovery [21-23]. About more than 50 percent of FDAapproved drugs were NPs or natural products derivatives [24,25]. Moreover, NPs have special selectivity to cellular targets [26]. Biologically active natural products would provide selective ligands for disease-related targets [27], and influence the disease-related pathways and eventually shift the biological network from disease status to the healthy status [28].

\section{Plant origin}

The use of natural products with therapeutic properties is as ancient as human civilisation and, for a long time, mineral, plant and animal products were the main sources of drugs [29]. About 25\% of the drugs prescribed worldwide come from plants, 121 such active compounds being in current use. Of the 252 drugs considered as basic and essential by the World Health Organisation (WHO), 11\% are exclusively of plant origin and a significant number are synthetic drugs obtained from natural precursors. Examples of important drugs obtained from plants are digoxin from Digitalis spp., quinine and quinidine from Cinchona spp., vincristrine and vinblastine from Catharanthus roseus, atropine from Atropa belladonna and morphine and codeine from Papaver somniferum [29]. It is estimated that $60 \%$ of anti-tumour and antiinfectious drugs already on the market or under clinical trial are of natural origin [30]. The vast majority of these cannot yet be synthesised economically and are still obtained from wild or cultivated plants. Natural compounds can be lead compounds, allowing the design and rational planning of new drugs, biomimetic synthesis development and the discovery of new therapeutic properties not yet attributed to known compounds. In addition, compounds such as muscarine, physostigmine, cannabinoids, yohimbine, forskolin, colchicines and phorbol esters, all obtained from plants, are important tools used in pharmacological, physiological and biochemical studies [29,31].

The use of natural plant products has been reported as antistress, for growth promotion, appetite stimulation, tonic and immunostimulation, and to have aphrodisiac and antimicrobial properties in finfish and shrimp larviculture due to the presence of active principle components such as alkaloids, flavanoids, pigments, phenolics, terpenoids, steroids, and essential oils [32-38]. Herbs, rich sources of immuneenhancing substances, are used in many countries to promote health, increase the body's natural resistance to infection and in prevention and treatment of various diseases [39]. Herbal products are cheaper source for therapeutics and have greater accuracy compared with chemotherapeutic agents, and offer a viable solution for all the problems which aquaculture faces today. Curcuma longa contains curcumene, turmerone, and turmerol [40]. Curcuminoids and gingerols have been reported to have antimicrobial, antifungal, anti-inflammatory, and antioxidant activities [41]. Curcuma longa has several components with immunomodulatory and antioxidant properties [39,42,43]. Ocimum sanctum has a vast number of therapeutic applications, such as in cardiopathy, homeopathy, leucoderma, asthma, bronchitis, catarrhal fever, hepatopathy, nausea, lumbago, hiccups, and skin diseases; the aqueous leaf extract also demonstrates antibacterial activity [44]. Curcumin essential oil from C. longa seeds has anti-inflammatory [45], antioxidant [43], antimicrobial [46], antibacterial [47], and antiulcer [48] properties. Azadirachta indica aqueous extracts of stem bark increase the production of lymphocytic migration inhibition factor in man [49]; its purified extract contains immunomodulators. Aqueous leaf extract possesses potent immunostimulant activity [50] that significantly decreases blood sugar level and prevents adrenaline and glucose-induced hyperglycemia [51]. Azadirachtin has possible uses as an immunostimulant or an adjuvant in vaccination that stimulates and enhances primary and secondary immune response in Mozambique tilapia Oreochromis mossambicus [52]. Enhanced primary and secondary antibody responses have been observed in Oreochromis mossambicus administered with azadirachtin, derived from the seed kernel of A. indica [52].

An extensive search for studies in which herbal medicines were used to treat fish parasites was conducted, and the data from this search have been presented in Table 3 [53]. These data may help future researchers in selecting herbal medicines to deepen the research and development studies on new products [53]. It can be seen that herbal medicines against I. multifiliis parasites and various species of monogeneans have been widely studied. This is mainly because of the great importance of these fish parasites in aquaculture worldwide and the lack of safe treatments.

\section{Animal origin}

Specific substances may be taken from animals and used as drugs. Hormones and other endocrine products such as epinephrine and insulin may be taken as examples although they are not presently being

\begin{tabular}{|c|c|c|c|c|}
\hline \multirow{3}{*}{ S. No. } & \multicolumn{4}{|c|}{ Mode of application } \\
\hline & \multicolumn{4}{|c|}{ Immersion } \\
\hline & Name of drug & $\begin{array}{l}\text { Commercial } \\
\text { name }\end{array}$ & Recommended dose & Indications for use \\
\hline 1 & Chloramine-T & $\begin{array}{l}\text { HALAMID® } \\
\text { Aqua - NADA } \\
141-423\end{array}$ & $\begin{array}{l}\text { Freshwater-reared salmonids: } \\
12 \text { to } 20 \text { milligrams per liter water in a continuous flow water supply or as a } \\
\text { static bath once per day for } 60 \text { minutes on consecutive or alternative days for } \\
\text { three treatments } \\
\text { Walleye: } \\
10 \text { to } 20 \text { milligrams per liter water in a continuous flow water supply or as a } \\
\text { static bath once per day for } 60 \text { minutes on consecutive or alternative days for } \\
\text { three treatments } \\
\text { Freshwater-reared warmwater finfish: } \\
20 \text { milligrams per liter water in a continuous flow water supply or as a static } \\
\text { bath once per day for } 60 \text { minutes on consecutive or alternative days for three } \\
\text { treatments }\end{array}$ & $\begin{array}{l}\text { For the control of mortality in freshwater- } \\
\text { reared salmonids due to bacterial gill disease } \\
\text { associated with Flavobacterium spp. } \\
\text { For the control of mortality in walleye due to } \\
\text { external columnaris disease associated with } \\
\text { Flavobacterium columnare } \\
\text { For the control of mortality in freshwater- } \\
\text { reared warmwater finfish due to external } \\
\text { columnaris disease associated with } \\
\text { Flavobacterium columnare }\end{array}$ \\
\hline
\end{tabular}


Citation: Kumar V, Roy S (2017) Aquaculture Drugs: Sources, Active Ingredients, Pharmaceutic Preparations and Methods of Administration. J Aquac Res Development 8: 510. doi: 10.4172/2155-9546.1000510

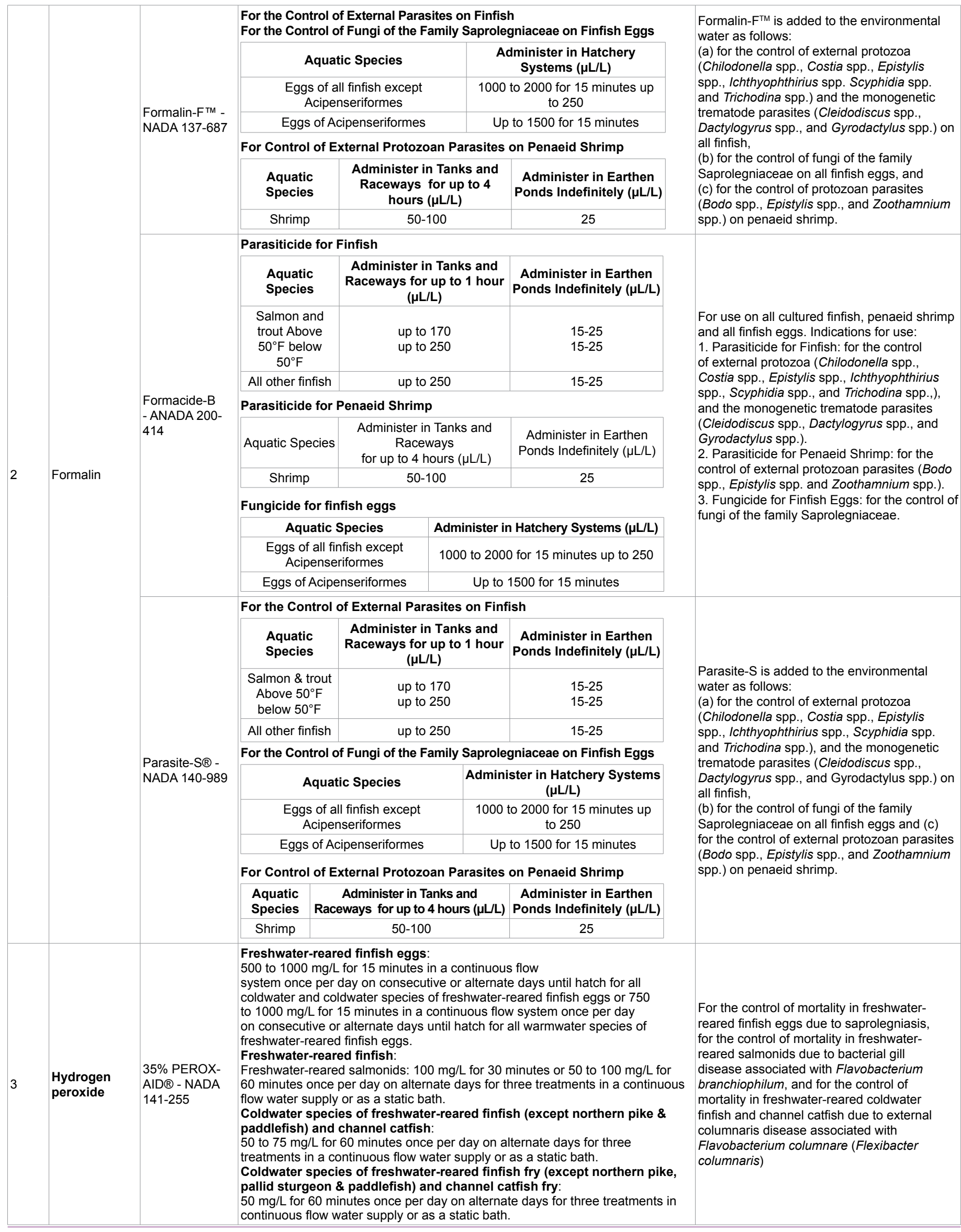


Citation: Kumar V, Roy S (2017) Aquaculture Drugs: Sources, Active Ingredients, Pharmaceutic Preparations and Methods of Administration. J Aquac Res Development 8: 510. doi: 10.4172/2155-9546.1000510

Page 4 of 13

\begin{tabular}{|c|c|c|c|c|c|c|c|c|}
\hline \multirow{5}{*}{4} & \multirow{5}{*}{$\begin{array}{l}\text { Oxytetracy- } \\
\text { cline hydro- } \\
\text { chloride }\end{array}$} & $\begin{array}{l}\text { Oxymarine }^{\mathrm{TM}}- \\
\text { NADA } 130-435\end{array}$ & \multicolumn{5}{|c|}{$\begin{array}{l}200 \text { to } 700 \mathrm{mg} \text { oxytetracycline hydrochloride (buffered)/liter of water for } 2 \text { to } 6 \\
\text { hours for finfish/fry and fingerling by immersion route of administration. } \\
\text { (Amount of Active Ingredient: } 1 \text { gram per } 2.73 \text { grams of powder) }\end{array}$} & $\begin{array}{l}\text { For the marking of skeletal tissues in finfish } \\
\text { fry and fingerlings as an aid in identification. }\end{array}$ \\
\hline & & $\begin{array}{l}\text { Oxytetracycline } \\
\text { HCl Soluble } \\
\text { Powder-343- } \\
\text { ANADA 200- } \\
247\end{array}$ & \multicolumn{5}{|c|}{$\begin{array}{l}200 \text { to } 700 \mathrm{mg} \text { oxytetracycline/L of water for } 2-6 \mathrm{hrs} \text { for finfish/fry and fingerling } \\
\text { by immersion route of administration. } \\
\text { (Amount of Active Ingredient: } 272.2 \mathrm{~g} \text { packet contains } 204.8 \mathrm{~g} \text { oxytetracycline } \\
\mathrm{HCl} \text {; } \\
135.5 \mathrm{~g} \text { packet contains } 102.4 \mathrm{~g} \text { oxytetracycline } \mathrm{HCl} \text { ) }\end{array}$} & $\begin{array}{l}\text { For the marking of skeletal tissues in finfish } \\
\text { fry and fingerlings as an aid in identification. }\end{array}$ \\
\hline & & $\begin{array}{l}\text { PENNOX } 343 \\
\text { - ANADA } 200- \\
026\end{array}$ & \multicolumn{5}{|c|}{$\begin{array}{l}\text { Immerse in } 200-700 \text { milligrams of oxytetracycline hydrochloride (buffered)/L } \\
\text { of water for } 2-6 \text { hours for finfish/fry and fingerling by immersion route of } \\
\text { administration. } \\
\text { (Amount of Active Ingredient: Each } 23.9 \text { oz packet contains } 512 \text { grams of } \\
\text { oxytetracycline hydrochloride). }\end{array}$} & $\begin{array}{l}\text { For the marking of skeletal tissues in finfish } \\
\text { fry and fingerlings as an aid in identification. }\end{array}$ \\
\hline & & $\begin{array}{l}\text { Terramycin } 343 \\
\text { (oxytetracycline } \\
\text { HCl) Soluble } \\
\text { Powder - NADA } \\
008-622\end{array}$ & \multicolumn{5}{|c|}{$\begin{array}{l}200 \text { to } 700 \mathrm{mg} \text { oxytetracycline } \mathrm{HCl} / \mathrm{L} \text { of water for } 2-6 \mathrm{hrs} \text { for finfish/fry and } \\
\text { fingerling by immersion route of administration. } \\
\text { (Amount of Active Ingredient: } 4.78 \text { oz packet contains } 102.4 \mathrm{~g} \text { oxytetracycline } \\
\mathrm{HCl} \text {; } \\
9.55 \mathrm{oz} \text { packet contains } 204.8 \mathrm{~g} \text { oxytetracycline } \mathrm{HCl} \text {; } \\
4.5 \mathrm{lb} \text { tub contains } 1543.5 \mathrm{~g} \text { oxytetracycline } \mathrm{HCl} \text { ). }\end{array}$} & $\begin{array}{l}\text { For the marking of skeletal tissues in finfish } \\
\text { fry and fingerlings. }\end{array}$ \\
\hline & & $\begin{array}{l}\text { Tetroxy Aquatic } \\
- \text { ANADA 200- } \\
460\end{array}$ & \multicolumn{5}{|c|}{$\begin{array}{l}200-700 \mathrm{mg} \text { oxytetracycline hydrochloride (buffered)/liter of water for 2-6 hours } \\
\text { depending on species by immersion route of administration. } \\
\text { (Amount of Active Ingredient: Oxytetracycline hydrochloride } 366 \mathrm{mg} / \mathrm{g} \text { of } \\
\text { powder). }\end{array}$} & $\begin{array}{l}\text { To mark skeletal tissues, most often the } \\
\text { otoliths, of all finfish fry and fingerlings for } \\
\text { subsequent identification. }\end{array}$ \\
\hline \multirow[t]{3}{*}{5} & $\begin{array}{l}\text { Tricaine meth- } \\
\text { anesulfonate }\end{array}$ & $\begin{array}{l}\text { Tricaine-S } \\
\text { - ANADA 200- } \\
226\end{array}$ & \multicolumn{5}{|c|}{$\begin{array}{l}\text { The product is available in the form of a crystalline powder to be mixed in water } \\
\text { for immersion of the animal. } \\
\text { The route of administration is immersion of the animal at levels ranging from } 10 \\
\text { to } 1,000 \mathrm{mg} / \text { liter. }\end{array}$} & $\begin{array}{l}\text { Tricaine-S is intended for the temporary } \\
\text { immobilization of fish, amphibians, and } \\
\text { other aquatic, cold-blooded animals. It has } \\
\text { been recognized as a valuable tool for the } \\
\text { proper handling of these animals during } \\
\text { manual spawning (fish stripping), weighing, } \\
\text { measuring, marking, surgical operations, } \\
\text { transport, photography, and research. }\end{array}$ \\
\hline & \multicolumn{8}{|r|}{ 年 } \\
\hline & Name of drug & $\begin{array}{l}\text { Commercial } \\
\text { name }\end{array}$ & \multicolumn{5}{|l|}{ Recommended dose } & Indications for use \\
\hline \multirow{11}{*}{\multicolumn{2}{|c|}{$\begin{array}{l}\text { Chorionic } \\
\text { gonadotropin }\end{array}$}} & \multirow{11}{*}{$\begin{array}{l}\text { Chorulon® - } \\
\text { NADA 140-927 }\end{array}$} & \multicolumn{5}{|c|}{$\begin{array}{l}\text { Should be administered intramuscularly, depending on the fish species. } \\
\text { - A dose of } 50 \text { to } 510 \text { I.U. per pound body weight (BW) for males and } \\
\text { - } 67 \text { to } 1816 \text { I.U. per pound BW for females, for one to three injections. }\end{array}$} & \multirow{11}{*}{$\begin{array}{l}\text { Chorulon }{ }^{\circledR} \text { (chorionic gonadotropin) is } \\
\text { indicated as an aid in improving spawning } \\
\text { function in male and female brood finfish. }\end{array}$} \\
\hline & & & \multirow{2}{*}{$\begin{array}{l}\text { Nomenclature } \\
\text { Common name, } \\
\text { Genus, Species, } \\
\text { Family }\end{array}$} & \multicolumn{2}{|c|}{$\begin{array}{l}\text { Dose/Dose Range } \\
\text { (I.U./lb BW/injection) }\end{array}$} & \multirow[t]{2}{*}{$\begin{array}{l}\text { Number of } \\
\text { Injections }\end{array}$} & \multirow[t]{2}{*}{$\begin{array}{l}\text { Injection } \\
\text { Interval } \\
\text { (hours) }\end{array}$} & \\
\hline & & & & Males & Females & & & \\
\hline & & & $\begin{array}{l}\text { Yellow perch, Perca } \\
\text { flavescens, Percidae }\end{array}$ & $\mathrm{nt}^{*}$ & $67-300$ & 1 & - & \\
\hline & & & $\begin{array}{l}\text { Striped bass, } \\
\text { Morone saxitilis, } \\
\text { Percichthyidae }\end{array}$ & $50-500$ & $75-252$ & 1 & - & \\
\hline & & & $\begin{array}{l}\text { White bass, } \\
\text { Morone chrysops, } \\
\text { Percichthyidae }\end{array}$ & $65-510$ & $91-750$ & 1 & - & \\
\hline & & & $\begin{array}{l}\text { Razorback sucker, } \\
\text { Xyrauchen texanus, } \\
\text { Catostomidae }\end{array}$ & $\mathrm{nt}^{*}$ & 100 & 3 & 24 & \\
\hline & & & $\begin{array}{c}\text { Walleye, Stizostedion } \\
\text { vitreum, Percidae }\end{array}$ & $75-400$ & $145-830$ & $1-3$ & 72 & \\
\hline & & & $\begin{array}{l}\text { Red snapper, } \\
\text { Lutjanus } \\
\text { campechanus, } \\
\text { Lutjanidae }\end{array}$ & 250 & 500 & 1 & - & \\
\hline & & & $\begin{array}{l}\text { Sauger, Stizostedion } \\
\text { canadense, Percidae }\end{array}$ & 500 & $500-1000$ & 1 & - & \\
\hline & & & $\begin{array}{l}\text { Chinese catfish, } \\
\text { Clarius fuscus, } \\
\text { Clariidae }\end{array}$ & $\mathrm{nt}^{*}$ & 1816 & 1 & - & \\
\hline & \multicolumn{8}{|c|}{ Medicated Articles/Feeds } \\
\hline & Name of drug & $\begin{array}{l}\text { Commercial } \\
\text { name }\end{array}$ & \multicolumn{5}{|l|}{ Recommended dose } & Indi \\
\hline
\end{tabular}


Citation: Kumar V, Roy S (2017) Aquaculture Drugs: Sources, Active Ingredients, Pharmaceutic Preparations and Methods of Administration. J Aquac Res Development 8: 510. doi: 10.4172/2155-9546.1000510

\begin{tabular}{|c|c|c|c|c|}
\hline 1 & \multirow[b]{5}{*}{ Florfenicol } & $\begin{array}{l}\text { Aquaflor }{ }^{\circledR} \text { - } \\
\text { NADA 141-246 }\end{array}$ & $\begin{array}{l}10 \mathrm{mg} \text { of florfenicol per } \mathrm{kg} \text { of body weight for } 10 \text { consecutive days for catfish by } \\
\text { oral via feed. } \\
\text { (Amount of Active Ingredients: } 500 \mathrm{~g} \text { of florfenicol per } \mathrm{kg} \text { ). }\end{array}$ & $\begin{array}{l}\text { For the control of mortality in catfish due to } \\
\text { enteric septicemia of catfish associated with } \\
\text { Edwardsiella ictaluri. }\end{array}$ \\
\hline & & $\begin{array}{l}\text { Aquaflor® - } \\
\text { NADA 141-246 }\end{array}$ & $\begin{array}{l}10 \mathrm{mg} \text { florfenicol/kg of fish/day for } 10 \text { consecutive days for freshwater-reared } \\
\text { salmonids via oral } \\
\text { (Amount of Active Ingredients: } 500 \mathrm{~g} \text { of florfenicol per } \mathrm{kg}(227.27 \mathrm{~g} \mathrm{per} \mathrm{lb)).}\end{array}$ & $\begin{array}{l}\text { For the control of mortality in freshwater- } \\
\text { reared salmonids due to coldwater } \\
\text { disease associated with Flavobacterium } \\
\text { psychrophilum. }\end{array}$ \\
\hline & & $\begin{array}{l}\text { Aquaflor }{ }^{\circledR} \text { - } \\
\text { NADA } 141-246\end{array}$ & $\begin{array}{l}10 \mathrm{mg} \text { florfenicol } / \mathrm{kg} \text { of fish/day for } 10 \text { consecutive days to freshwater-reared } \\
\text { salmonids via oral } \\
\text { (Amount of Active Ingredients: } 500 \mathrm{~g} \text { of florfenicol per kg (227.27 g per lb)). }\end{array}$ & $\begin{array}{l}\text { For the control of mortality due to furunculosis } \\
\text { associated with Aeromonas salmonicida. }\end{array}$ \\
\hline & & $\begin{array}{l}\text { Aquaflor }{ }^{\circledR}- \\
\text { NADA 141-246 }\end{array}$ & $\begin{array}{l}\text { Freshwater-reared warmwater finfish: } 10-15 \mathrm{mg} / \mathrm{kg} \text { of fish/day for } 10 \\
\text { consecutive days via oral } \\
\text { Other freshwater-reared finfish: } 10 \mathrm{mg} / \mathrm{kg} \text { of fish/day for } 10 \text { consecutive days } \\
\text { via oral } \\
\text { (Amount of Active Ingredients: } 500 \mathrm{~g} \text { of florfenicol per } \mathrm{kg}(227.27 \mathrm{~g} \text { per lb)). }\end{array}$ & $\begin{array}{l}\text { Freshwater-reared warmwater finfish: For } \\
\text { the control of mortality due to streptococcal } \\
\text { septicemia associated with Streptococcus } \\
\text { iniae. } \\
\text { Freshwater-reared finfish: For the control } \\
\text { of mortality due to columnaris disease } \\
\text { associated with Flavobacterium columnare. }\end{array}$ \\
\hline & & $\begin{array}{l}\text { Aquaflor }{ }^{\circledR}- \\
\text { NADA } 141-246\end{array}$ & $\begin{array}{l}10-15 \mathrm{mg} / \mathrm{kg} \text { body weigh/day for } 10 \text { consecutive days to freshwater-reared } \\
\text { finfish, freshwater-reared warmwater finfish, freshwater-reared salmonids, } \\
\text { catfish } \\
\text { (Amount of Active Ingredients: } 500 \mathrm{~g} \text { of florfenicol per kg ( } 227.27 \mathrm{~g} \mathrm{per} \mathrm{lb)).}\end{array}$ & $\begin{array}{l}\text { Catfish: For the control of mortality due to } \\
\text { enteric septicemia of catfish associated with } \\
\text { Edwardsiella ictaluri. } \\
\text { Freshwater-reared salmonids: For the } \\
\text { control of mortality due to coldwater } \\
\text { disease associated with Flavobacterium } \\
\text { psychrophilum. } \\
\text { Freshwater-reared salmonids: For the control } \\
\text { of mortality due to furunculosis associated } \\
\text { with Aeromonas salmonicida. } \\
\text { Freshwater-reared warmwater finfish: For } \\
\text { the control of mortality due to streptococcal } \\
\text { septicemia associated with Streptococcus } \\
\text { iniae. } \\
\text { Freshwater-reared finfish: For the control } \\
\text { of mortality due to columnaris disease } \\
\text { associated with Flavobacterium columnare. }\end{array}$ \\
\hline 2 & $\begin{array}{l}\text { Oxytetracy- } \\
\text { cline dihy- } \\
\text { drate }\end{array}$ & $\begin{array}{l}\text { Terramycin }{ }^{\circledR} \\
200 \text { for Fish - } \\
\text { NADA } 038-439\end{array}$ & $\begin{array}{l}\text { Salmonids and catfish }-2.5 \text { to } 3.75 \mathrm{~g} \text { oxytetracycline } / 100 \mathrm{lb} \text { of fish for } 10 \text { days } \\
\text { via oral feed. } \\
\text { Lobsters }-1 \mathrm{~g} \text { oxytetracycline/lb of medicated feed administered as the sole } \\
\text { ration for } 5 \text { consecutive days via oral feed. } \\
\text { (Amount of Active Ingredients: } 200 \mathrm{~g} \text { oxytetracycline/lb). }\end{array}$ & $\begin{array}{l}\text { Salmonids - Control of ulcer disease caused } \\
\text { by Hemophilus piscium, furunculosis } \\
\text { caused by Aeromonas salmonicida, } \\
\text { bacterial hemorrhagic septicemia caused by } \\
\text { Aeromonas liquefaciens, and pseudomonas } \\
\text { disease. } \\
\text { Catfish - Control of bacterial hemorrhagic } \\
\text { septicaemia caused by Aeromonas } \\
\text { liquefaciens and pseudomonas disease. } \\
\text { Lobsters - Control of gaffkemia caused by } \\
\text { Aerococcus viridans. }\end{array}$ \\
\hline \multirow[t]{2}{*}{3} & \multirow{2}{*}{$\begin{array}{l}\text { Sulfadi- } \\
\text { methoxine/ } \\
\text { ormetoprim }\end{array}$} & $\begin{array}{l}\text { Romet-30® - } \\
\text { NADA } 125-933\end{array}$ & $\begin{array}{l}50 \mathrm{mg} / \mathrm{kg} \text { of body weight per day for the maximum } 5 \text { day treatment period via } \\
\text { oral feed. }\end{array}$ & $\begin{array}{l}\text { For treatment of bacterial infectious diseases } \\
\text { of salmonids caused by Aeromonas } \\
\text { salmonicida (furunculosis) and by Yersinia } \\
\text { ruckeria (enteric red mouth). }\end{array}$ \\
\hline & & $\begin{array}{l}\text { Sulfamerazine } \\
\text { - NADA 033- } \\
950\end{array}$ & \multicolumn{2}{|l|}{ Original approval in 1967 - not currently marketed } \\
\hline
\end{tabular}

Table 2: Aquaculture drugs approved by the Food and Drug Administration (USA).

used in the treatment of fish disease [54]. Animals as drug sources for fish medication are extremely rare; very few animal - derived fish drugs have been developed and even fewer are in use. Moreover, there is risk of infection with various pathogens and diseases are involved from the use of animal-derived substances in drug manufacturing. Therefore, the efforts of the drug manufacturer to correspond to regulatory actions and to implement authoritative guidelines should be improved [55].

\section{Chemical substances}

Technically, all drugs are chemical substances but what is intended here is to include all those chemicals used as drugs that are not obtained from living resources. Some such as the inorganic acids, bases, salts and oxides are extremely simple compounds owing their action to the presence of a single atom or ion [54]. Many are of mineral origin, in which a rock or its pulverized equivalent is used as is. Still others are extremely complex chemicals, synthesized entirely in the laboratory with absolutely no counterpart in nature, e.g. the sulfonamides. Petroleum and coal furnish the hydrocarbon bases for many of these drugs, regardless of whether they are finalized though distillation or chemical synthesis. One building block of special note, from which a great many organic compounds are obtained by chemical substitution are those, both occurring and synthesized that utilize the benzene ring 
Citation: Kumar V, Roy S (2017) Aquaculture Drugs: Sources, Active Ingredients, Pharmaceutic Preparations and Methods of Administration. J Aquac Res Development 8: 510. doi: 10.4172/2155-9546.1000510

Page 6 of 13

\begin{tabular}{|c|c|c|c|c|c|c|c|c|}
\hline \multirow[b]{2}{*}{ Parasite } & \multirow[b]{2}{*}{ Host } & \multicolumn{2}{|l|}{ Phytotherapic } & \multicolumn{5}{|c|}{ Most effective treatment } \\
\hline & & $\begin{array}{l}\text { Type/Active } \\
\text { compound }\end{array}$ & Plant & Route & Period & Concentration & Results & Reference \\
\hline $\begin{array}{l}\text { Argulus spp. } \\
\text { (Argulidae) }\end{array}$ & $\begin{array}{l}\text { Carassius auratus } \\
\text { (Cyprinidae) }\end{array}$ & Piperine & Piper longum & Water & $48 \mathrm{~h}$ & $9.0 \mathrm{mg} / \mathrm{L}$ & $\begin{array}{l}100 \% \text { efficacy } \\
\text { compared to control }\end{array}$ & $\begin{array}{l}\text { Kumar et al. } \\
{[87]}\end{array}$ \\
\hline $\begin{array}{l}\text { Dactylogyrus } \\
\text { intermedius } \\
\text { (Dactylogyridae) }\end{array}$ & $\begin{array}{l}\text { Carassius auratus } \\
\text { (Cyprinidae) }\end{array}$ & $\begin{array}{l}\text { Osthol and } \\
\text { isopimpinellin }\end{array}$ & Fructus cnidii & Water & $48 \mathrm{~h}$ & $\begin{array}{l}\text { Osthol } 1.6 \mathrm{mg} / \mathrm{L} \text { and } \\
\text { isopimpinellin } \\
9.5 \mathrm{mg} / \mathrm{L}\end{array}$ & $\begin{array}{l}100 \% \text { efficacy } \\
\text { compared to control }\end{array}$ & $\begin{array}{l}\text { Wang et al. } \\
{[88]}\end{array}$ \\
\hline $\begin{array}{l}\text { Dactylogyrus } \\
\text { intermedius } \\
\text { (Dactylogyridae) }\end{array}$ & $\begin{array}{l}\text { Carassius auratus } \\
\text { (Cyprinidae) }\end{array}$ & $\begin{array}{l}\text { Methanol extract } \\
\text { Aqueous extract }\end{array}$ & $\begin{array}{l}\text { Semen } \\
\text { aesculi }\end{array}$ & Water & $48 \mathrm{~h}$ & $\begin{array}{l}10 \mathrm{mg} / \mathrm{L} \\
12 \mathrm{mg} / \mathrm{L}\end{array}$ & $\begin{array}{l}100 \% \text { efficacy } \\
\text { compared to control } \\
100 \% \text { efficacy } \\
\text { compared to control }\end{array}$ & $\begin{array}{l}\text { Liu et al. } \\
{[89]}\end{array}$ \\
\hline $\begin{array}{l}\text { Dactylogyrus } \\
\text { intermedius } \\
\text { (Dactylogyridae) }\end{array}$ & $\begin{array}{l}\text { Carassius auratus } \\
\text { (Cyprinidae) }\end{array}$ & $\begin{array}{l}\text { Dioscin and } \\
\text { polyphyllin D }\end{array}$ & $\begin{array}{l}\text { Paris } \\
\text { polyphylla }\end{array}$ & Water & $48 \mathrm{~h}$ & $\begin{array}{l}\text { Dioscin EC50* }=0.44 \\
\mathrm{mg} / \mathrm{L} \text { and polyphyllin } \mathrm{D} \\
\mathrm{EC50}=0.70 \mathrm{mg} / \mathrm{L}\end{array}$ & $\begin{array}{l}\text { 1.5-3.0 times more } \\
\text { effective than the } \\
\text { positive control, } \\
\text { mebendazole }\end{array}$ & $\begin{array}{l}\text { Wang et al. } \\
{[90]}\end{array}$ \\
\hline $\begin{array}{l}\text { Dactylogyrus } \\
\text { intermedius } \\
\text { (Dactylogyridae) }\end{array}$ & $\begin{array}{l}\text { Carassius auratus } \\
\text { (Cyprinidae) }\end{array}$ & Gracillin & $\begin{array}{l}\text { Dioscorea } \\
\text { zingiberensis }\end{array}$ & Water & $48 \mathrm{~h}$ & $0.9 \mathrm{mg} / \mathrm{L}$ & $\begin{array}{l}\text { High antiparasitic } \\
\text { activity; almost } 10 \\
\text { times more effective } \\
\text { than the positive } \\
\text { control, mebendazole }\end{array}$ & $\begin{array}{l}\text { Wang et al. } \\
\text { [91] }\end{array}$ \\
\hline $\begin{array}{l}\text { Dactylogyrus } \\
\text { intermedius } \\
\text { (Dactylogyridae) }\end{array}$ & $\begin{array}{l}\text { Carassius auratus } \\
\text { (Cyprinidae) }\end{array}$ & Sanguinarine & $\begin{array}{l}\text { Macleaya } \\
\text { microcarpa }\end{array}$ & Water & $48 \mathrm{~h}$ & $0.7 \mathrm{mg} / \mathrm{L}$ & $\begin{array}{l}100 \% \text { efficacy } \\
\text { compared to control }\end{array}$ & $\begin{array}{l}\text { Wang et al. } \\
{[92]}\end{array}$ \\
\hline $\begin{array}{l}\text { Dactylogyrus } \\
\text { intermedius } \\
\text { (Dactylogyridae) }\end{array}$ & $\begin{array}{l}\text { Ctenopharyngodon } \\
\text { idella } \\
\text { (Cyprinidae) }\end{array}$ & Chelerythrine & $\begin{array}{l}\text { Chelidonium } \\
\text { majus }\end{array}$ & Water & $48 \mathrm{~h}$ & $1.60 \mathrm{mg} / \mathrm{L}$ & $\begin{array}{l}100 \% \text { efficacy } \\
\text { compared to control }\end{array}$ & Li et al. [93] \\
\hline $\begin{array}{l}\text { Dactylogyrus } \\
\text { intermedius } \\
\text { (Dactylogyridae) }\end{array}$ & $\begin{array}{l}\text { Carassius auratus } \\
\text { (Cyprinidae) }\end{array}$ & Osthole & $\begin{array}{l}\text { Radix } \\
\text { angelicae } \\
\text { pubescentis }\end{array}$ & Water & $48 \mathrm{~h}$ & $1.6 \mathrm{mg} / \mathrm{L}$ & $\begin{array}{l}100 \% \text { efficacy } \\
\text { compared to control }\end{array}$ & $\begin{array}{l}\text { Wang et al. } \\
{[90]}\end{array}$ \\
\hline $\begin{array}{l}\text { Dactylogyrus } \\
\text { intermedius } \\
\text { (Dactylogyridae) }\end{array}$ & $\begin{array}{l}\text { Carassius auratus } \\
\text { (Cyprinidae) }\end{array}$ & $\begin{array}{l}\text { Bruceine } A \text { and } \\
\text { bruceine } D\end{array}$ & $\begin{array}{l}\text { Brucea } \\
\text { javanica }\end{array}$ & Water & $48 \mathrm{~h}$ & $1 \mathrm{mg} / \mathrm{L}$ & $\begin{array}{l}\text { Bruceine } A=97 \% \\
\text { efficacy and bruceine } \\
D=91.2 \% \text { efficacy; } \\
2-2.5 \text { times more } \\
\text { effective than the } \\
\text { positive control, } \\
\text { mebendazole }\end{array}$ & $\begin{array}{l}\text { Wang et al. } \\
[91])\end{array}$ \\
\hline $\begin{array}{l}\text { Dactylogyrus } \\
\text { intermedius } \\
\text { (Dactylogyridae) }\end{array}$ & $\begin{array}{l}\text { Carassius auratus } \\
\text { (Cyprinidae) }\end{array}$ & Methanol extract & $\begin{array}{l}\text { Radix } \\
\text { Bupleuri } \\
\text { chinensis }\end{array}$ & Water & $48 \mathrm{~h}$ & $10 \mathrm{mg} / \mathrm{L}$ & $\begin{array}{l}100 \% \text { efficacy } \\
\text { compared to control }\end{array}$ & $\begin{array}{l}\text { Wu et al. } \\
{[94]}\end{array}$ \\
\hline $\begin{array}{l}\text { Dactylogyrus } \\
\text { intermedius } \\
\text { (Dactylogyridae) }\end{array}$ & $\begin{array}{l}\text { Carassius auratus } \\
\text { (Cyprinidae) }\end{array}$ & $\begin{array}{l}\text { Aqueous extract } \\
\text { Aqueous extract }\end{array}$ & $\begin{array}{l}\text { Cinnamomum } \\
\text { cassia }\end{array}$ & Water & $48 \mathrm{~h}$ & $\begin{array}{l}30 \mathrm{mg} / \mathrm{L} \\
40 \mathrm{mg} / \mathrm{L}\end{array}$ & $\begin{array}{l}100 \% \text { efficacy } \\
\text { compared to control } \\
100 \% \text { efficacy } \\
\text { compared to control }\end{array}$ & Ji et al. [95] \\
\hline $\begin{array}{l}\text { Dactylogyrus } \\
\text { intermedius } \\
\text { (Dactylogyridae) }\end{array}$ & $\begin{array}{l}\text { Carassius auratus } \\
\text { (Cyprinidae) }\end{array}$ & Methanol extract & $\begin{array}{l}\text { Dryopteris } \\
\text { crassirhizoma }\end{array}$ & Water & $48 \mathrm{~h}$ & $60 \mathrm{mg} / \mathrm{L}$ & $\begin{array}{l}100 \% \text { efficacy } \\
\text { compared to control }\end{array}$ & Lu et al. [96] \\
\hline $\begin{array}{l}\text { Gyrodactylus } \\
\text { spp. } \\
\text { (Gyrodactylidae) }\end{array}$ & $\begin{array}{l}\text { Gasterosteus } \\
\text { aculeatus } \\
\text { (Gasterosteidae) }\end{array}$ & Essential oil & $\begin{array}{l}\text { Melaleuca } \\
\text { alternifolia }\end{array}$ & Water & $48 \mathrm{~h}$ & 30 ppmv & $\begin{array}{l}90 \% \text { efficacy compared } \\
\text { to control }\end{array}$ & $\begin{array}{l}\text { Steverding } \\
\text { et al. [97] }\end{array}$ \\
\hline $\begin{array}{l}\text { Gyrodactylus } \\
\text { elegans and } \\
\text { Dactylogyrus } \\
\text { extensus } \\
\text { (Gyrodactylidae \& } \\
\text { Dactylogyridae) } \\
\end{array}$ & $\begin{array}{l}\text { Carassius auratus } \\
\text { (Cyprinidae) }\end{array}$ & Methanol extract & $\begin{array}{l}\text { Piper } \\
\text { guineense }\end{array}$ & Water & $96 \mathrm{~h}$ & $1.5 \mathrm{mg} / \mathrm{L}$ & $\begin{array}{l}\text { Approx. } 45 \% \text { efficacy } \\
\text { against Dactylogyrus } \\
\text { and } 75 \% \text { against } \\
\text { Gyrodactylus } \\
\text { compared to control }\end{array}$ & $\begin{array}{l}\text { Ekanem et al. } \\
{[98]}\end{array}$ \\
\hline $\begin{array}{l}\text { Gyrodactylus } \\
\text { turnbulli } \\
\text { (Gyrodactylidae) }\end{array}$ & $\begin{array}{l}\text { Poecilia reticulate } \\
\text { (Poeciliidae) }\end{array}$ & Freeze-dried & $\begin{array}{l}\text { Allium } \\
\text { sativum }\end{array}$ & Water & $\begin{array}{l}\text { One } \\
\text { time }\end{array}$ & $0.03 \mathrm{mg} / \mathrm{L}$ & $\begin{array}{l}95 \% \text { efficacy } \\
\text { compared to control }\end{array}$ & $\begin{array}{l}\text { Schelkle } \\
\text { et al. [99] }\end{array}$ \\
\hline $\begin{array}{l}\text { Ichthyophthirius } \\
\text { multifiliis } \\
\text { (Ichthyophthiriidae) }\end{array}$ & $\begin{array}{l}\text { Carassius auratus } \\
\text { (Cyprinidae) }\end{array}$ & $\begin{array}{l}\text { Methanol extract } \\
\text { Petroleum-ether } \\
\text { extract }\end{array}$ & $\begin{array}{l}\text { Mucuna } \\
\text { pruriens } \\
\text { Carica } \\
\text { papaya }\end{array}$ & Water & $\begin{array}{l}72 \mathrm{~h} \\
96 \mathrm{~h}\end{array}$ & $\begin{array}{l}200 \mathrm{mg} / \mathrm{L} \\
250 \mathrm{mg} / \mathrm{L}\end{array}$ & $\begin{array}{l}92 \% \text { (skin) and } 91 \% \\
\text { (gill) efficacy; Fish } \\
\text { mortality decreased } \\
45 \% \text { compared to } \\
\text { control } \\
92 \% \text { efficacy (skin); } \\
\text { Fish mortality } \\
\text { decreased } 30 \% \\
\text { compared to control }\end{array}$ & $\begin{array}{l}\text { Ekanem et al. } \\
{[100]}\end{array}$ \\
\hline $\begin{array}{l}\text { Ichthyophthirius } \\
\text { multifiliis } \\
\text { (Ichthyophthiriidae) }\end{array}$ & $\begin{array}{l}\text { Ctenopharyngodon } \\
\text { idella } \\
\text { (Cyprinidae) }\end{array}$ & Sanguinarine & $\begin{array}{l}\text { Macleaya } \\
\text { cordata }\end{array}$ & Water & $48 \mathrm{~h}$ & $0.9 \mathrm{mg} / \mathrm{L}$ & $\begin{array}{l}96.8 \% \text { efficacy (gill) } \\
\text { compared to control }\end{array}$ & $\begin{array}{l}\text { Yao et al. } \\
{[101]}\end{array}$ \\
\hline
\end{tabular}


Citation: Kumar V, Roy S (2017) Aquaculture Drugs: Sources, Active Ingredients, Pharmaceutic Preparations and Methods of Administration. J Aquac Res Development 8: 510. doi: 10.4172/2155-9546.1000510

Page 7 of 13

\begin{tabular}{|c|c|c|c|c|c|c|c|c|}
\hline $\begin{array}{l}\text { Ichthyophthirius } \\
\text { multifiliis } \\
\text { (Ichthyophthiriidae) }\end{array}$ & $\begin{array}{l}\text { Squaliobarbus } \\
\text { curriculus } \\
\text { (Cyprinidae) }\end{array}$ & $\begin{array}{l}\text { Dihydrosanguinarine } \\
\text { and } \\
\text { dihydrochelerythrine }\end{array}$ & $\begin{array}{l}\text { Macleaya } \\
\text { microcarpa }\end{array}$ & Water & $48 \mathrm{~h}$ & $\begin{array}{l}\text { Dihydrosanguinarine } \\
5.18 \mathrm{mg} / \mathrm{L} \text { and } \\
\text { Dihydrochelerythrine } \\
9.43 \mathrm{mg} / \mathrm{L}\end{array}$ & $\begin{array}{l}\text { Both showed high } \\
\text { antiparasitic activity } \\
\text { (EC50*: } 5.19 \text { and } 9.43 \\
\text { mg/L, respectively) }\end{array}$ & $\begin{array}{l}\text { Yao et al. } \\
\text { [102] }\end{array}$ \\
\hline $\begin{array}{l}\text { Ichthyophthirius } \\
\text { multifiliis } \\
\text { (Ichthyophthiriidae) }\end{array}$ & $\begin{array}{l}\text { Carassius auratus } \\
\text { (Cyprinidae) }\end{array}$ & Aqueous extract & $\begin{array}{l}\text { Capsicum } \\
\text { frutescens }\end{array}$ & Water & $4 \mathrm{~h}$ & $1: 32$ and $1: 64$ (v:v) & $\begin{array}{l}\text { Decreases parasite } \\
\text { prevalence }(13.3 \% \\
\text { and } 40 \%, \text { respectively) } \\
\text { compared to } 100 \% \text { in } \\
\text { control }\end{array}$ & $\begin{array}{l}\text { Ling et al. } \\
{[103]}\end{array}$ \\
\hline $\begin{array}{l}\text { Ichthyophthirius } \\
\text { multifiliis } \\
\text { (Ichthyophthiriidae) }\end{array}$ & $\begin{array}{l}\text { Carassius auratus } \\
\text { (Cyprinidae) }\end{array}$ & Methanol extract & $\begin{array}{l}\text { Magnolia } \\
\text { officinalis } \\
\text { and } \\
\text { Sophora } \\
\text { alopecuroides }\end{array}$ & Water & $1 \mathrm{~h}$ & $\begin{array}{l}\text { M. officinalis } 40 \mathrm{mg} / \mathrm{L} \\
\text { and } \mathrm{S} \text {. alopecuroides } \\
320 \mathrm{mg} / \mathrm{L}\end{array}$ & $\begin{array}{l}\text { Decreases tomont } \\
\text { survival }(24.7 \% \\
\text { and } 44.7 \% \text {, } \\
\text { respectively) compared } \\
\text { to } 91.7 \% \text { in the control }\end{array}$ & Yi et al. [104] \\
\hline $\begin{array}{l}\text { Ichthyophthirius } \\
\text { multifiliis } \\
\text { (Ichthyophthiriidae) }\end{array}$ & $\begin{array}{l}\text { Ictalurus punctatus } \\
\text { (Ictaluridae) }\end{array}$ & Pentagalloylglucose & $\begin{array}{l}\text { Galla } \\
\text { chinensis }\end{array}$ & Water & 10 days & $20 \mathrm{mg} / \mathrm{L}$ & $\begin{array}{l}93.3 \% \text { survival of } \\
\text { infected catfish } \\
\text { compared to } 0 \% \text { in the } \\
\text { control }\end{array}$ & $\begin{array}{l}\text { Zhang et al. } \\
{[105]}\end{array}$ \\
\hline $\begin{array}{l}\text { Ichthyophthirius } \\
\text { multifiliis } \\
\text { (Ichthyophthiriidae) }\end{array}$ & $\begin{array}{l}\text { Carassius auratus } \\
\text { (Cyprinidae) }\end{array}$ & $\begin{array}{l}\text { Chelerythrine } \\
\text { and chloroxylonine }\end{array}$ & $\begin{array}{l}\text { Toddalia } \\
\text { asiatica }\end{array}$ & Water & $72 \mathrm{~h}$ & $\begin{array}{l}1.8 \mathrm{mg} / \mathrm{L} \text { chelerythrine } \\
\text { and } 8 \mathrm{mg} / \mathrm{L} \\
\text { chloroxylonine }\end{array}$ & $\begin{array}{l}\text { Decreases parasite } \\
\text { prevalence compared } \\
\text { to control ( } 50 \% \text { and } \\
60 \% \text {, respectively) }\end{array}$ & $\begin{array}{l}\text { Shan et al. } \\
{[106]}\end{array}$ \\
\hline $\begin{array}{l}\text { Ichthyophthirius } \\
\text { multifiliis } \\
\text { (Ichthyophthiriidae) }\end{array}$ & $\begin{array}{l}\text { Piaractus } \\
\text { mesopotamicus } \\
\text { (Serrasalmidae) }\end{array}$ & Essential oil & $\begin{array}{l}\text { Melaleuca } \\
\text { alternifolia }\end{array}$ & Water & $\begin{array}{l}2 \mathrm{~h} / \\
\text { day for } \\
5 \text { days }\end{array}$ & $50 \mu \mathrm{l} / \mathrm{L}$ & $\begin{array}{l}99.8 \% \text { (skin) and } \\
98.8 \% \text { (gill) efficacy; } \\
56.33 \% \text { fish survival } \\
\text { compared to } 0 \% \text { in the } \\
\text { control }\end{array}$ & $\begin{array}{l}\text { Valladao et } \\
\text { al. [107] }\end{array}$ \\
\hline Monogenea & $\begin{array}{l}\text { Heterobranchus } \\
\text { longifilis } \\
\text { (Clariidae) }\end{array}$ & Ethanol extract & $\begin{array}{l}\text { Artemisia } \\
\text { annua }\end{array}$ & Water & $1 \mathrm{~h}$ & $200 \mathrm{mg} / \mathrm{L}$ & $\begin{array}{l}85 \% \text { efficacy } \\
\text { compared to control }\end{array}$ & $\begin{array}{l}\text { Ekanem and } \\
\text { Brisibe [108] }\end{array}$ \\
\hline $\begin{array}{l}\text { Myxobolus sp. } \\
\text { (Myxobolidae) }\end{array}$ & $\begin{array}{l}\text { Diplodus puntazzo } \\
\text { (Sparidae) }\end{array}$ & Essential oil & $\begin{array}{l}\text { Origanum } \\
\text { minutiflorum }\end{array}$ & $\begin{array}{l}\text { Oral } \\
\text { (Feed) }\end{array}$ & 35 days & $\begin{array}{l}8 \mathrm{~mL} 5 / \mathrm{Kg} \\
\text { of biomass }\end{array}$ & $\begin{array}{l}\text { Decreases parasite } \\
\text { prevalence ( } 37 \text { to } 39 \% \\
\text { compared to control) } \\
\text { and infestation intensity }\end{array}$ & $\begin{array}{l}\text { Karagouni } \\
\text { et al. [109] }\end{array}$ \\
\hline $\begin{array}{l}\text { Neobenedenia sp. } \\
\text { (Capsalidae) }\end{array}$ & $\begin{array}{l}\text { Lates calcarifer } \\
\text { (Latidae) }\end{array}$ & Allicin & $\begin{array}{l}\text { Allium } \\
\text { sativum }\end{array}$ & Water & $1 \mathrm{~h}$ & $15.2 \mu \mathrm{l} / \mathrm{L}$ & $\begin{array}{l}\text { Decreased significantly } \\
\text { hatching (approx. } 90 \% \\
\text { compared to control } \\
\text { group) and longevity of } \\
\text { oncomiracidium }\end{array}$ & $\begin{array}{l}\text { Militz } \\
\text { et al. [110] }\end{array}$ \\
\hline $\begin{array}{l}\text { Pseudodactylogyrus } \\
\text { (Pseudodactylogyridae) }\end{array}$ & $\begin{array}{l}\text { Anguilla anguilla } \\
\text { (Anguillidae) }\end{array}$ & $\begin{array}{l}\text { Ginkgolic acid } \\
\text { C13:0 and C15:1 }\end{array}$ & Ginkgo biloba & Water & $48 \mathrm{~h}$ & $\begin{array}{l}\text { C13:0 }(2.5 \mathrm{mg} / \mathrm{L}) \\
\text { and C15:1 }(6.0 \mathrm{mg} / \mathrm{L})\end{array}$ & $\begin{array}{l}100 \% \text { efficacy } \\
\text { compared to control } \\
\text { group }\end{array}$ & $\begin{array}{l}\text { Wang } \\
\text { et al. [111] }\end{array}$ \\
\hline $\begin{array}{l}\text { Trichodina sp. } \\
\text { (Trichodinidae) }\end{array}$ & $\begin{array}{l}\text { Oreochromis } \\
\text { niloticus } \\
\text { (Cichlidae) }\end{array}$ & Crude extract & $\begin{array}{l}\text { Allium } \\
\text { sativum and } \\
\text { Terminalia } \\
\text { catappa }\end{array}$ & Water & $48 \mathrm{~h}$ & $800 \mathrm{mg} / \mathrm{L}$ & $\begin{array}{l}\text { Decreases } 100 \% \text { of the } \\
\text { parasites on the skin } \\
\text { and fins }\end{array}$ & $\begin{array}{l}\text { Chitmanat } \\
\text { et al. [112] }\end{array}$ \\
\hline $\begin{array}{l}\text { Trichodina sp. } \\
\text { (Trichodinidae) }\end{array}$ & $\begin{array}{l}\text { Oreochromis } \\
\text { niloticus } \\
\text { (Cichlidae) }\end{array}$ & Aqueous extract & $\begin{array}{l}\text { Camellia } \\
\text { sinensis }\end{array}$ & Water & $5 \mathrm{~min}$ & $0.9 \%$ & $\begin{array}{l}\text { Decreases } 95 \% \text { of the } \\
\text { parasites on the skin } \\
\text { and fins }\end{array}$ & El-Deen [113] \\
\hline $\begin{array}{l}\text { Trichodina sp. and } \\
\text { Gyrodactilus sp. } \\
\text { (Trichodinidae \& } \\
\text { Gyrodactylidae) }\end{array}$ & $\begin{array}{l}\text { Oreochromis } \\
\text { niloticus } \\
\text { (Cichlidae) }\end{array}$ & $\begin{array}{l}\text { Crushed garlic } \\
\text { cloves }\end{array}$ & $\begin{array}{l}\text { Allium } \\
\text { sativum }\end{array}$ & Water & $\begin{array}{l}\text { indefi- } \\
\text { nite }\end{array}$ & $300 \mathrm{mg} / \mathrm{L}$ & $\begin{array}{l}\text { Reduced } 23 \% \text { of } \\
\text { parasitized fish } \\
\text { compared to control } \\
\text { group }\end{array}$ & $\begin{array}{l}\text { Abd El- } \\
\text { Galil and } \\
\text { Aboelhadid } \\
{[114]}\end{array}$ \\
\hline $\begin{array}{l}\text { Uronema sp. } \\
\text { (Chaetophoraceae) }\end{array}$ & $\begin{array}{l}\text { Pampus argenteus } \\
\text { (Stromateidae) }\end{array}$ & Essential oil & $\begin{array}{l}\text { Melaleuca } \\
\text { alternifolia }\end{array}$ & Water & $\begin{array}{l}30 \mathrm{~min} / \\
\text { day for } \\
5 \text { days }\end{array}$ & 20 ppm & $\begin{array}{l}\text { Healing of skin } \\
\text { lesions }\end{array}$ & $\begin{array}{l}\text { Al-Yaqout } \\
\text { and Azad } \\
{[115]}\end{array}$ \\
\hline
\end{tabular}

Table 3: Plant-derived antimicrobial compounds with potential usefulness for treating parasitic diseases in aquaculture.

[55]. Disinfectants are widely used in many spheres of aquaculture. They are used both in hatchery and grow-out systems mainly for equipment cleaning, to maintain hygiene and in some cases to treat disease (Table 4) $[56]$.

\section{Synthetic drugs}

Synthetic drugs are manufactured to pharmacologically resemble naturally occurring drugs. Antimicrobial drugs may have different types of chemical structures, and they act on different parts of bacterial machinery [57]. In general, antibiotics work by one of two mechanisms: (a) bactericidal effect, i.e., the antibiotic generally kills the bacteria by interfering with either the formation of the bacterium's cell wall or its cell contents. Examples include penicillin, fluoroquinolones, and metronidazole. (b) bacteriostatic effect, i.e., the antibiotic stops bacteria from multiplying by interfering with bacterial protein production, DNA replication, or other aspects of bacterial cellular metabolism. Examples include tetracyclines, sulfonamides, chloramphenicol, and macrolides [57].

Some of the antibiotics that inhibit bacterial cell wall synthesis include Beta-lactams (penicillins, cephalosporins) and glycopeptides. Beta-Lactam drugs block the synthesis of the bacterial cell wall by interfering with the enzymes required for the synthesis of the 
Citation: Kumar V, Roy S (2017) Aquaculture Drugs: Sources, Active Ingredients, Pharmaceutic Preparations and Methods of Administration. J Aquac Res Development 8: 510. doi: 10.4172/2155-9546.1000510

Page 8 of 13

\begin{tabular}{|l|l|l|}
\hline Trade Name & Active ingredient & Dose \\
\hline EDTA & Sodium thiosulfate & $0.1-1 \mathrm{ppm}$ \\
\hline Bleaching powder & Chlorine & $60 \mathrm{ppm}$ \\
\hline Timsen & n-alkyl dimethyl benzyl ammonium chloride + stabilized urea & $20 \mathrm{~g} / 33 \mathrm{dec}$. (For prevention) $80 \mathrm{~g} / 33 \mathrm{dec}$. (For Treatment) \\
\hline Emsen & n-alkyl dimethyl benzyl ammonium chloride + stabilized urea & $80 \mathrm{~g} / 33 \mathrm{dec}$. \\
\hline Water clear & Sodium Thiosulphate & In case of 5-6 feet deep water body 2-3 L/100 dec. \\
\hline Pathocide & Benzyl chronium + Natural polymer of glucosamine & $200 \mathrm{ml} / 33 \mathrm{dec}$. \\
\hline Omicide & Benzyl ammonium chloride+ urea & $200 \mathrm{ml} / 33 \mathrm{dec}$. after 24 hours $150 \mathrm{ml}$ \\
\hline Lenocide & Alkyl benzyl dimethyl ammonium chloride + Poly-2-deoxy-2 Amino glucose & $500-1000 \mathrm{ml} /$ acre \\
\hline Microdine-lodine 20\% & Nonyl Alkyl Phenoxy Poly Ethylene Oxide lodine Complex & $2-2.5 \mathrm{~L} / \mathrm{acre}$ \\
\hline Formalin & $38 \%$ formaldehyde & $1-3 \mathrm{ppm}$ \\
\hline BKC & Benzalkonium chloride solution & Spread with water, $0.5 \mathrm{ppm}$ \\
\hline Efinol & Efinol & $5-8 \mathrm{~g} / 1000$ liter water \\
\hline *1 acre=0.40-hectare, 1 Decimal (dec.) $=0.004$ hectares & \\
\hline
\end{tabular}

Table 4: Chemicals used as disinfectant.

\begin{tabular}{|l|l|l|}
\hline \multicolumn{2}{|l|}{ Mechanism of action of antibacterial agents } & Example of antibacterial agents \\
\hline Interference with cell wall synthesis & $\begin{array}{l}\text { Beta-lactam } \\
\text { Glycopeptides }\end{array}$ & $\begin{array}{l}\text { Cephalosporins, carbapenems, monobactams } \\
\text { Vancomycin, teicoplanin }\end{array}$ \\
\hline Protein synthesis inhibition & $\begin{array}{l}\text { Bind to 50S ribosomal subunit } \\
\text { Bind to 30S ribosomal subunit }\end{array}$ & $\begin{array}{l}\text { Macrolides, chloramphenicol, clindamycin, linezolid, quinupristin- } \\
\text { dalfopristin } \\
\text { Aminoglycosides, tetracyclines }\end{array}$ \\
\hline Interference with nucleic acid synthesis & $\begin{array}{l}\text { Bind to bacterial isoleucyl-tRNA synthetase } \\
\text { Inhibit DNA synthesis } \\
\text { Inhibit RNA synthesis }\end{array}$ & $\begin{array}{l}\text { Mupirocin } \\
\text { Fluoroquinolones } \\
\text { Rifampin }\end{array}$ \\
\hline Inhibition of metabolic pathways & Sulfonamides, folic acid analogues \\
\hline Disruption of bacterial membrane structures & Polymyxins, deptomycin \\
\hline
\end{tabular}

Table 5: Mechanisms of action of antibiotics.

peptidoglycan layer. In contrast, some antibiotics inhibiting protein synthesis include macrolides, aminoglycosides, tetracyclines and chloramphenicol. These antibacterial drugs take advantage of the structural differences between bacterial and eukaryotic ribosomes to selectively inhibit bacterial growth. Macrolides, aminoglycosides, and tetracyclines bind to the $30 \mathrm{~S}$ subunit of the ribosome, whereas chloramphenicol binds to the 50S subunit (Table 5) [57].

\section{Active Ingredients}

A substance or compound that is intended to be used in the manufacture of a pharmaceutical product are generally called therapeutically active compound (ingredient). From a crude form in a plant or animal source, a drug of highly refined purity may be extracted which is much more potent and reliable than the original form. The extraction is termed the active principle of the drug [54]. Such drugs may be roughly classified into various groups or families according to their chemical structures.

\section{Alkaloids}

Alkaloids (literally - alkali like) are organic based nitrogenous compounds obtained most exclusively from plants (Table 3 ), although they may be found in animals [54]. Following the initial discoveries and isolations there was a gradual increase in the number of known and medicinally used alkaloids. Currently, the Dictionary of Natural Products (DNP) lists over 27,000 compounds as alkaloids [58]. As with natural products as a whole, many have proposed differing classificatory schemes for alkaloids. One popular scheme divides the whole class of compounds into three categories: (a) True alkaloids (compounds which derive from amino acid and a heterocyclic ring with nitrogen, (b) Protoalkaloids (compounds, in which the $\mathrm{N}$ atom derived from an amino acid is not a part of the heterocycle), and (c) Pseudoalkaloids (compounds, the basic carbon skeletons of which are not derived from amino acids) [59].
In the last 200-year period, many alkaloids became critical components of the global pharmaceutical armamentarium, and tremendous healing has resulted from their clinical application [60]. Buckingham [61] identified a total of 53 alkaloids used currently or within the last 50 years for pharmaceutical applications. To date less than $0.002 \%(53 / 27,000)$ of alkaloids or alkaloid-based drugs are marketed for such uses internationally. It is not surprising that such a diverse set of natural products and their derivatives yield medicines which are used in a variety of applications. However, in the last 25 years only galanthamine and taxol were newly introduced into biomedicine, and the former in essence through an extension of the therapeutic claims [62]. There are only less than 200 others which are commonly used in industrial processes and the manufacturing of commercial goods (for example: $\mathrm{N}, \mathrm{N}^{\prime}$-dioctadecanoylethanediamine is an antifoaming agent used in the polymer industry and methylamine hydrochloride is used in the tanning industry) [63].

\section{Glycosides}

Glycosides (glucosides) are plant derivatives containing a sugar and one or more other substances, which are usually steroids [54]. They may be broken down by hydrolysis and the nonessential carbohydrates (sugar) may be discarded to liberate the most active ingredient which is termed an aglycone or aglucone. Secondary metabolites, which have vital environmental and allelopathic functions for a host, and long tradition of ethnopharmacological applications preceding modern medicinal use, often occur in their native state as glycosides [64]. This however, may relieve the drug of some of its potency which is present in the parent glycoside molecule. The glycosides are usually colorless and are extracted from the plant with water and alcohol. They are named so that they should end in '-im'. Steroids are similar to sterols which are solid in room temperature. Thus, they may need to be dissolved in a transport medium of some sort or else administered orally. Glycosides exert a strong stimulant action on the heart and other areas of the body [54]. 


\section{Plant organic acids}

Plant organic acids have many similarities in action to inorganic acids. They are obtained naturally from the fruits of higher plants and are the cause of sour taste in fruits. Limes, lemons, grapefruits, oranges and other citrus plants contain citric acid [54]. Citric acid is a natural preservative, benign cleaning agent, antioxidant and adds sour taste to foods. Tartaric acid occurs chiefly in grapes and is made from cream of tartar deposits which coat the sides of wine vats during the fermentation process. If the fermentation process is allowed to extend too long, then another plant acid i.e., acetic acid may be produced. The discovery that grapefruit juice could alter drug metabolism was the serendipitous result of using the juice as part of a placebo preparation in a drug test conducted in Canada [65]. The drug felodipine was being evaluated for interactions with alcohol. Alcohol did affect the way the drug functioned, resulting in more side effects. The plasma concentrations of the drug in the placebo group that had received grapefruit juice rather than alcohol were surprisingly high. The same researchers then performed a follow-up study [65] using either grapefruit juice or orange juice; the grapefruit juice increased the bioavailability of the drug nifedipine by an average of $284 \%$ and orange juice had no such effect, indicating that it was a particular component of grapefruit juice that was responsible for this marked effect [66]. Plant organic acids are all characterized by the presence in their chemical structure of the $-\mathrm{COOH}$ radical. They are not extensively used in fish therapeutics although they may be very effective when application is made as antiseptics or disinfectants [54].

\section{Pharmaceutical Preparations}

The forms of drugs available are many and varied. Often the form in which a drug is compounded is merely a matter of convenience to the manufacturer or user. Other times it is a necessary form for administration. Knowing the difference and limitation of various types of formulations may mean the difference between success or failure in the treatment of a fish or of an epizootic [54]. The art and science of preparing drugs for medicinal use is called Pharmacy, thus a pharmaceutic preparation is the form in which a drug is made ready for use. Preparations may occur in the form of solids, liquids or gases. They may be intended for internal (systemic) or external use in water. The form in which a medication is prepared may serve as an indication of the manner in which it may be used [54]. For instance, capsules are generally (but not always) intended for internal use, while aqueous solutions are intended for introduction into the water for external use.

Today by the time that fish medicine has come upon the scene, the manufacturers themselves prepare the forms of medication in more or less predetermined dosages and in a variety of forms. This has simplified pharmacy to the extent that only the proper dosage needs to be weighed or counted out and dispensed. This makes the administration of drugs a great deal simpler and much more reliable than it was in the last century. It has led to greater uniformity and better quality with less danger of under or over dosage.

\section{Types of Solutions}

The aquatic nature of the fish environment and the universal use of baths as a form of treatment make solutions the prime form of medication in fish therapeutics. In fish pharmacology we use a working definition that a true solution is an aqueous preparation of a nonvolatile drug. Thus, a non-aqueous substance must be dissolved in another substance which in turn dissolved in water before there is a true solution [54]. Solutions consist of two parts: (a) the solute, which is the substance that is to be dissolved and (b) the solvent, which is the substance onto which the solute is dissolved and the base, aqueous or otherwise of the solution. Both solutes and solvents may be wither, solid, liquid or gas. The solubility of a substance that is the extent or degree to which a solute may be dissolved in a solvent is determined by a number of chemical and physical factors. Under some conditions a supersaturated solution may be obtained but this has no application in fish therapeutics.

\section{Aqueous preparation}

While virtually every drug or medication used in fish therapeutics must be an aqueous preparation in its final analysis, not all aqueous preparations are used in fish medicine. The most important preparation in this grouping is liquors are commonly called solutions (Table 3). Solutions are merely non-volatile substances dissolved in water. They constitute the vast majority of fish preparation available. Other solid or powdered forms used in baths are ultimately solutions in pond or aquarium into which fish is placed [54].

An insoluble substance such as many of the salts, oxides and hydroxides of calcium and other compounds may form colloidal suspension called magmas. Magmas are fine pharmaceutical suspensions in which the suspensoid has a high degree of physical attraction to the aqueous vehicle, forming a gelatinous mixture that maintains that uniformity and stability of the suspension. Magmas are administered orally [67]. Infusion are obtained by boiling or steeping vegetable materials such as bark, roots or seed in water to obtain an aqueous raw extract of a medicinal agent. They are generally unreliable as obtaining a proper dosage is concerned.

\section{Alcoholic preparation}

In general ethyl alcohol is the one understood when an alcoholic preparation is mentioned. Other types of alcohols are usually stated in the drug title if one of them is used. Other alcohols commonly employed as solvents for alcoholic preparations include methyl and isopropyl [54].

Tinctures are alcoholic or partially alcoholic solutions of nonvolatile extracts of vegetable or animal origin drugs or of pure chemical compounds [68]. Tinctures vary in strength but usually do not exceed $10 \%$ active principle in strength. In general, they are used topically as bacteriostats and disinfectants. They are usually being somewhat unstable solution, and many will precipitate in strong light and dark bottles for storage are advised. Tincture of iodine is used frequently and may serve as an example but as a group, tinctures are little used in fish therapeutics. Fluid extract are concentrated liquid alcoholic solutions, sometimes reaching $100 \%$ in active strength [69]. They are obtained by percolation of a vegetable drug so that one millilitre of fluid extract contains one gram of drug (Table 3 ).

Acetone is used to produce a solution of quinaldine for anesthetization of fishes [70]. However, its use is not condoned because of its irritating properties on sensitive membranes, i.e., fish gills. A much more rational substitute to acetone is ethyl alcohol, which has anesthetizing properties of their own.

\section{Solid preparations}

The most basic solid preparation is a crystalline or powdery substance of either synthetic or natural origin which is usually of very high quality and purity. Such drug substances or mixture of substances when finely ground to a more or less uniform grade, are termed powders and constitute the base of all solid drug preparations. They are the most economical when dispensed in bulk but may come in a variety 
of forms, either with or without filler substances. Powder forms are the most suitable medium for transporting large volume of a drug. They are then simply mixed with water to produce an aqueous solution that is ready to use [71]. An exact measured quantity of powdered drug in a sealed sterile container to which a suitable solvent usually water can be added is termed as sterile powder. Many injectible antibiotic drugs which do not have a stable shelf life after being mixed (reconstituted) are marketed in this fashion. Ampicillin is an example of a drug that can occurs primarily as sterile powder [54]. Powder may be placed in combination with lactose or some other inert binders moistened with alcohol or some other volatile liquid to form a wet mass and then placed into a suitable mold and compressed into a tablet. While powdered drug forms are more suitable as well as more economical for use in treating fishes, tablets come in more exact premeasured doses ready for use without the necessity of being weighed out which is an important consideration when scales are not at hand or technical expertise is lacking [72].

Tablets have largely been replaced by drug manufacturers with capsules. Capsules are exact premeasured quantities of a drug enclosed in a gelatine capsule. They may be either hard or soft but are usually soluble in water after only a few minutes of immersion [54]. Capsules are more or less commercial formulation of pills. Sometimes capsules are coated with indigestible substances like salol or steric acid or else may be made with a substance that is indigestible in acid medium of the stomach but will dissolve for release and absorption into the system in the intestine [72].

\section{Methods of Administration}

The fundamental principle of treating fishes is the same as for any other animal. However, the variable nature of the fluid habitat of fishes introduces some special problems and consideration into their therapeutics [54]. Their poikilothermic nature must also be considered when searching for the proper drug and its route of administration. Many otherwise desirable drugs may be rendered inactive or otherwise unsuitable for use because of these considerations [73].

\section{Topical applications}

The simplest and most direct method of treating fishes is to apply the drug directly to the body surfaces. Wound, skin ulceration and localized infections or traumas may be treated by this method by using a concentrated solution of a suitable chemical usually an oxidizing agent, disinfectant or antiseptic [74]. A great many drugs in the form of emulsion, linaments, ointments and lotion which would otherwise be highly useful agents are unsuitable for use in on fishes because they will either wash off, dissolve or float to the water surface because of their lighter specific gravity. In order to be useful topically, a drug must be insoluble in water, act immediately upon contact, be denser than water or be adhesive enough to adhere to the body of a fish [75].

In the use of this method of treatment, the individual fish must be caught and raised to the surfaces in order for the drug to be applied. A wet towel thrown over the fish will allow both a firm grip to hold the fish steady and also reduce dehydration or drying out if the fish kept out of water for any length of time [73]. Application can be made by pouring the drug directly onto the body surfaces or from a squeeze drop bottle or it can be applied with a cotton swab on the end of a small dowel. Moreover, this is not an economically desirable method for treating large group of fishes because of the time and effort involved [54].

\section{Administration by bath}

Virtually every drug available is water soluble compound designed to be added to the aquarium. Moreover, the commercial fish farmers and hatchery operators has somewhat wider range of choice with oral and injection medication however, he is still bound to the ease and lower cost of administration (Table 3) [75]. Only the veterinarian and some public aquariums have full range of techniques and drugs available however, the most commonly used forms of treatment are bath $[73,74]$. Baths are equally applicable whether for one fish in a small aquarium or a full fish farm. The enormous number of fish pathogen either external parasite or bacterial infective agents can be best treated through bath medication because of ease of absorption into the fish system through the fish gills, mucous membrane and integuments (in case of systemic bacterial infection) [75].

Bath as treatment may be divided into a variety of types depending on the concentration of the drug or chemical used and types of pathogen being combated [76]. Technically, baths may be considered as an extended form of topical application. They are classified according to time duration ranging from a momentary dip to an indefinite period in which the drug is applied and then simply left in the water [77]. Some precaution and consideration must be taken in case of bath medication. Various combinations of drugs and chemicals must be avoided as they might not only nullify the good effect of each other but also may become toxic to fish in their combined effect (commonly called drug antagonism) [54]. The $\mathrm{pH}$ of water must also be considered as some drugs become more or less efficient or lose their effect entirely at different $\mathrm{pH}$, while other may simply precipitate out of solution [73]. Some drugs may be inactivated by light and hence must be store in dark conditions. Water temperature and hardness has also a drastic effect on the activity of a drug or chemical, so care should be taken to maintain the efficacy of a drug or chemical against aquatic pathogens [75].

\section{Oral administration}

The oral administration of medication can save much time and effort when utilized in the treatment and in hatcheries and pond culture, where large numbers of fish are involved medicated feed are extensively used to combat systemic infections as a prophylaxis and to stimulate growth in food fishes (Table 3). However, there are some major drawbacks/ limitations which must not be overlooked or underestimated in oral administration [78,79]. On a smaller scale or individually a rubber tube catheter can be used feed and/ give exact dosage to sick fish that no longer have any appetite. This limited method is used primarily on large, expensive aquarium fish and on breeders in pond culture. To avoid the stress of handling to sick fish the catheter method of treatment is considered an efficient method of providing drug to valuable fishes [80].

Medicated feed in pellet form either of floating or sinking variety are thrown upon the surface of the water a process termed broadcasting feeding. This result is an uneven distribution of the feed among the fishes as the fish that are weaker or less aggressive receive little or no medication, while the healthy and aggressive ones probably obtain an overdose of the drug applied [81]. This may not harm in case of nontoxic antihelminthic drugs however, it is not good practice when using an antibiotic. In addition, the social hierarchy allows only those at the top to obtain the bulk of any food available, while those on the bottom must settle for left over. So, it is generally conceded that feeding medication to fish is most useful as a prophylaxis rather than as a treatment as we have discussed this may be more damaging in the long run [82]. Still, there are occasions when it may be the only way to save any appreciable number of fish population i.e., when for some reason a pond cannot be drained or during acute attack of bacterial hemorrhagic septicaemia, where speed of administrating a drug over an extensive 
area to an extensive population may be the only workable solution [81]. One other method of medicating fish orally especially marine fish is to administer the drug in the form of a bath of high salinity. Then, because of the natural osmotic forces, which cause marine fish to lose body fluids to its environment, it must drink seawater to replenish its losses. In doing so the fish takes in and utilizes the drugs in the water as well [54].

\section{Parenteral (injection) administration}

The metabolic processes of fish are associated with their poikilothermic nature. Their heart muscle and associated chambers do not pump blood reliably or strongly as it happens in advanced vertebrates. Their muscle tissue is poorly vascularised, which slowdowns the diffusion into the system [54]. It is the most effective and direct route of drug administration as long as consideration on action of drug is mentioned i.e., its absorption rate into various tissues and its exsorption route in fish. The methods of administration must be chosen carefully based upon an understanding of the drug being used [83].

However, the major limitation of Parenteral (injection) administration are the amount of time and effort involved in catching and injecting large number of fish as well as the tremendous stress that handling can place upon them [73]. Parenteral administration is best suited for small number of valuable fishes such as the routine injection of brooders Cyprinidae in the spring to prevent the occurrence of diseases (bacterial haemorrhagic septicaemia or furunculosis in Salmonidae). The treatment of valuable aquarium fishes is admirably suited to this approach provided the correct dose is used [84]. Some types of injection of special use in higher forms of life, i.e., intrapleural or intrasternal are impossible in fish because of the absence associated or comparable structures in fish. Intravenous injections are also both difficult and impractical on any but the largest fishes [85].

Parenteral administration of drug may be accomplished in a variety of ways, depending upon the drug to be used and its most desirable route of administration. In all cases, however injection is accomplished through the use of standard medical hypodermic syringe with hollow needles attached [54]. The size of syringe is determined by the amount of drug to be used, and the bore and length of the needle are determined by the size of the fish is to be used upon. Generally, doses are extremely small even in larger fish, and a tuberculin syringe with about 24 to 26 -gauge needle is probably the most commonly employed size (although a 30 -gauge needle can be used on extremely small fish) $[83,86]$.

\section{Conclusion and Future Perspectives}

Aquaculture has rapidly growing to a major industry, providing not only economic income and high-quality food products but also provides employment to hundreds of thousands of skilled and unskilled workers. Among different aquaculture practices, intensive fish culture helps in the development of stressful condition for aquatic animals which resulted in spread of microbial infection and diseases. The presence of disease in farmed fish populations has severe implications on both the affected fish and unaffected fish. Moreover, considering the rapid growth and importance of the aquaculture industry in many regions of the world, the widespread use of antibacterial agents is getting increased and intensified, and often unregulated (without professional consultation or supervision) for aquatic animal production, which has created many health issue problems, not only for fish but also for humans. Therefore, more research is needed in order to determine the consequences of the application of large quantities of antimicrobials, also, safer, more effective medicines are necessary, along with improvements in husbandry and management which will reduce the need for those medicaments.

\section{Acknowledgements}

Authors are thankful to Director, ICAR-Central Inland Fisheries Research Institute (CIFRI) for ample help and support. We are also thankful to central library of CIFRI for the same.

\section{Conflict of Interests}

All the authors here by certify that there is no conflict of interest regarding the publication of this article and with any financial issues.

\section{References}

1. FAO (2011) The State of World Fisheries and Aquaculture 2010. FAO Fisheries and Aquaculture Department. Rome, Italy.

2. Austin B, Austin DA (2007) Control in bacterial fish pathogens. Springer, The Netherlands.

3. Bergh $O$ (2007) The dual myths of the healthy wild fish and the unhealthy farmed fish. Dis Aquat Organ 75: 159-164.

4. Burka JF, Hammell KL, Horsberg TE, Johnson GR, Rainnie DJ, et al. (1997) Drugs in salmonid aquaculture - A review. Journal of Veterinary Pharmacology and Therapeutics 20: 333-349.

5. Defoirdt T, Sorgeloos $P$, Bossier $P$ (2011) Alternatives to antibiotics for the control of bacterial disease in aquaculture. Current Opinion in Microbiology 14 251-258.

6. Seo JS, Jeon EJ, Jung SH, Park MA, Kim NY (2015) Pharmacokinetics of amoxicillin trihydrate in cultured olive flounder (Paralichthys olivaceus). Journal of veterinary pharmacology and therapeutics 38: 86-92.

7. Sekkin S, Kum C (2011) Antibacterial drugs in fish farms: Application and its effects. Recent Advances in Fish Farms.

8. Phillips I, Casewell M, Cox T, De Groot B, Friis C, et al. (2004) Does the use of antibiotics in food animals pose a risk to human health? A critical review of published data. J Antimicrob Chemother 53: 28-52.

9. winton jr (2001) fish health Management. In: Fish Hatchery Management, American Fisheries Society, Bethesda, USA.

10. Lupin HM (2009) Human health aspects of drug and chemical use in aquaculture. In: The use of veterinary drugs and vaccines in Mediterranean aquaculture, CIHEAM - IAMZ

11. Sanders $P$ (2005) Antibiotic use in animals-Policies and control measures around Europe. In Antibiotic Policies, Springer US

12. Prescott JF (2008) Antimicrobial use in food and companion animals. Animal Health Research Reviews 9: 127-133.

13. Rodgers CJ, Furones MD (2009) Antimicrobial agents in aquaculture: Practice, needs and issues. In: The Use of Veterinary Drugs and Vaccines in Mediterranean Aquaculture, CIHEAM - IAMZ.

14. Smith PR, Le Breton A, Horsberg TE, Corsin F (2008) Guidelines fo antimicrobial use in aquaculture. In: Guide to Antimicrobial Use in Animals Oxford, UK: Blackwell Publishing Ltd.

15. FAO (2005) Responsible use of antibiotics in aquaculture. FAO Fisheries. Rome, Italy.

16. Shao ZJ (2001) Aquaculture pharmaceuticals and biologicals: current perspectives and future possibilities. Adv Drug Deliv Rev 50: 229-243.

17. Sapkota A, Sapkota AR, Kucharski M, Burke J, McKenzie S, et al. (2008) Aquaculture practices and potential human health risks: current knowledge and future priorities. Environ Int 34: 1215-1226.

18. Heuer OE, Kruse H, Grave K, Collignon P, Karunasagar I, et al. (2009) Human health consequences of use of antimicrobial agents in aquaculture. Clinical Infectious Diseases 49: 1248-1253.

19. Haskell SR, Payne MA, Webb Al, Riviere JE, Craigmill AL (2004) Current approved drugs for aquatic species. Journal of the American Veterinary Medical Association 224: 50-51.

20. Park ED, Lightner DV, Park DL (1994) Antimicrobials in shrimp aquaculture 
Citation: Kumar V, Roy S (2017) Aquaculture Drugs: Sources, Active Ingredients, Pharmaceutic Preparations and Methods of Administration. J Aquac Res Development 8: 510. doi: 10.4172/2155-9546.1000510

in the United States: regulatory status and safety concerns. Reviews of environmental contamination and toxicology 138: 1-20

21. Newman DJ, Cragg GM (2012) Natural products as sources of new drugs over the 30 years from 1981 to 2010 . Journal of Natural Products 75: 311-335.

22. Ji HF, Li XJ, Zhang HY (2009) Natural products and drug discovery can thousands of years of ancient medical knowledge lead us to new and powerfu drug combinations in the fight against cancer and dementia? Embo Reports 10: $194-200$

23. Harvey A (2008) Natural products in drug discovery. Drug Discovery Today 13: 894-901.

24. Kingston DGI (2011) Modern natural products drug discovery and its relevance to biodiversity conservation. Journal of Natural Products 74: 496-511.

25. Chin YW, Balunas MJ, Chai HB, Kinghorn AD (2006) Drug discovery from natural sources. The AAPS Journal 8: E239-E253.

26. Lagunin A, Filimonov D, Poroikov V (2010) Multi-targeted natural products evaluation based on biological activity prediction with pass. Current Pharmaceutical Design 16: 1703-1717.

27. Clardy J, Walsh C (2004) Lessons from natural molecules. Nature 432: 829837

28. Gu J, Gui Y, Chen L, Yuan G, Lu HZ, et al. (2013) Use of natural products as chemical library for drug discovery and network pharmacology. PLoS ONE 8 : e62839.

29. Rates SMK (2001) Plants as source of drugs. Toxicon 39: 603-613.

30. Yue-Zhong S (1998) Recent natural products based drug development: A pharmaceutical industry perspective. Journal of Natural Products 61: 10531071.

31. Williamson E, Okpako DT, Evans FJ (1996) Selection, preparation and pharmacological evaluation of plant material, Wiley, Chichester, USA.

32. Citarasu T, Babu MM, Punitha SMJ, Venket-Ramalingam K, Marian MP (2001) Control of pathogenic bacteria using herbal biomedicinal products in the larviculture system of Penaeus monodon. In: International conference on advanced technologies in fisheries and marine sciences, MS University, India.

33. Citarasu T (2010) Herbal biomedicines: A new opportunity for aquaculture industry. Aquacult Int 18: 403-414.

34. Sivaram V, Immanul G, Namita R, Murugan Y (2004) Influence of selected Indian stimulant herbs against white spot syndrome virus (WSSV) infection in black tiger shrimp, Penaeus monodon with reference to haematological, biochemical and immunological changes. Fish Shellfish Immunol 21: 372-384.

35. Kumar V, Roy S, Barman D (2015) Effect of Mikania cordata (Burm) BL Robins on Non-Specific Immune Response of Catla catla (Hamilton, 1822) Agains Aphanomyces invadans. Fishery Technology 52: 20-25

36. Kumar V, Kumar S, Pandey PK, Raman RP, Prasad KP, et al. (2014) Growth and haemato-immunological response to dietary I-carrageenan in Labeo rohita (Hamilton, 1822) juveniles. The Israeli Journal of Aquaculture Bamidgeh 66 : 971-981

37. Kumar V, Roy S, Meena DK, Sarkar UK (2016a) Application of probiotics in shrimp aquaculture: Importance, mechanisms of action, and methods of administration. Reviews in Fisheries Science \& Aquaculture 24: 342-368.

38. Kumar V, Roy S, Barman D (2016b) Effect of Mikania cordata on non-specific immune response and survival of Labeo rohita against Aphanomyces invadans. The Journal of Animal \& Plant Sciences 26: 1833-1842.

39. Devasagayam TPA, Sainis KB (2002) Immune system and antioxidants, especially those derived from Indian medicinal plants. Ind J Exp Biol 40: 639655.

40. Bhakuni DS, Dhar ML, Dhawan BN, Mehrotra BN (1969) Screening of Indian plants for biological activity, part II. Indian Journal of Experimental Biology 7 250-262.

41. Masuda T, Jitoe A (1994) Antioxidative and anti-inflammatory compounds from tropical gingers: isolation, structure determination, and activities of cassumunins $A, B$ and $C$, new complex curcuminoids from Zingiber cassumunar. Journal of Agricultural and Food Chemistry 42: 1850-1856.

42. Ruby AJ, Kuttan G, Babu KD, Rajasekharan KN, Kultan R (1995) Anti-tumour and antioxidant activity of natural curcuminoids. Cancer Letters 109: 79.
43. Selvam R, Subramanian I, Gayathri R, Angayarkanni N (1995) The anti-oxidant activity of turmeric (Curcuma longa). Journal of Ethnopharmacology 47: 59-63.

44. Gupta SK, Prakash J, Srivastava S (2002) Validation of traditional claim of tulsi, Ocimum sanctum Linn, as a medicinal plant. Indian Journal of Experimental Biology 40: 765-773.

45. Anto RJ, Kuttan G, Dinesh BKV, Rajasekharan KN, Kuttan R (1998) Antiinflammatory activity of natural and synthetic curcuminoids. Pharmacy and Pharmacology Communications 4: 103-105.

46. Janssen AM, Scheffer JJ, Ntezurubanza L, Svendsen AB (1989) Antimicrobial activities of some Ocimum species grown in Rwanda. Journal of Ethnopharmacology 26: 57-63.

47. Nagamura CV, Nakamura TU, Bando E, Fernandes A, Melo N, et al. (1999) Antibacterial activity of Ocimum gratissimum L. essential oil. Memorias do Instituto Oswaldo Cruz 94: 675-678.

48. Singh KP, Gupta AS, Pendse VK, Mahatma OP, Bhandari IS, et al. (1999) Experimental and clinical studies on Tinospora coradifolia. Indian Journal of Medical Research 10: 9-12.

49. Van der Nat JM, Klerx P, Dijk HV, De Silva KT, Labadie RP (1987) Immunomodulatory activity of an aqueous extract of Azadirachta indica stem bark. Journal of Ethnopharmacology 19: 125-131.

50. Sen P, Maiti PC, Puri S, Ray A (1992) Mechanism of antistress activity of Ocimum sanctum Linn, eugenol and Tinospora malabarica in experimental animals. Indian Journal of Experimental Biology 30: 596-604

51. Murty KS, Rao DN, Rao DK, Murty LBG (1978) A preliminary study on the hypoglycemic and antihyperglycemic effects of Azadirachta indica. Indian Journal of Pharmacology 10: 247-250.

52. Logambal SM, Michael RD (2001) Azadirachtin e an immunostimulant for Oreochromis mossambicus (Peters). J Aqua Trop 16: 339-347.

53. Valladao GMR, Gallani SU, Pilarski F (2015) Phytotherapy as an alternative for treating fish disease. Journal of veterinary pharmacology and therapeutics 38: $417-428$.

54. Herwig N (1979) Handbook of drugs and chemicals used in the treatment of fish diseases. Charles $\mathrm{C}$. Thomas.

55. Berger CN, Le Donne P, Windemann H (2005) Use of substances of anima origin in pharmaceutics and compliance with the TSE-risk guideline-a market survey. Biologicals 33: 1-7.

56. Faruk MAR, Ali MM, Patwary ZP (2008) Evaluation of the status of use of chemicals and antibiotics in freshwater aquaculture activities with specia emphasis to fish health management. Journal of the Bangladesh Agricultural University 6: 381-390.

57. Romero J, Feijoo CG, Navarrete P (2012) Antibiotics in aquaculture - Use, abuse and alternatives, health and environment in aquaculture.

58. Hocking G (1997) A Dictionary of Natural Products (1st edn) Plexus Pub Medford, NJ, USA

59. Eagleson M (1994) Concise Encyclopedia Chemistry (1st edn) Walter de Gruyter, Berlin

60. The Royal Society of Chemistry (1971) The Alkaloids, Specialist Periodical Reports, The Royal Society of Chemistry, London.

61. Buckingham J (2010) Dictionary of Alkaloids (1st ed.) CRC Press, Boca Raton, FL.

62. Heinrich M, Teoh HL (2004) Galanthamine from snowdrop - The development of a modern drug against Alzheimer's disease from local Caucasian knowledge. J Ethnopharmacol 92: 147-162.

63. Amirkia V, Heinrich M (2014) Alkaloids as drug leads-A predictive structura and biodiversity-based analysis. Phytochemistry Letters.

64. Grynkiewicz G, Wieslaw S, Boryski J (2008) Synthetic analogs of natural glycosides in drug discovery and development. Acta Pol Pharm 65: 655-676.

65. Bailey DG, Spence JD, Edgar B, Bayliff CD, Arnold JM (1989) Ethano enhances the hemodynamic effects of felodipine. Clin Invest Med 12: 357-362.

66. Dharmananda S (2001) The interactions of herbs and drugs. Institute for Traditional Medicine, Portland, Oregon.

67. Breuer E, Chorghade MS, Fischer J, Golomb G (2009) Glossary of terms related to pharmaceutics. Pure and Applied Chemistry 81: 971-999. 
Citation: Kumar V, Roy S (2017) Aquaculture Drugs: Sources, Active Ingredients, Pharmaceutic Preparations and Methods of Administration. J Aquac Res Development 8: 510. doi: 10.4172/2155-9546.1000510

68. De Oliveira CC, Abud AP, De Oliveira SM, De Guimaraes SF, De Andrade LF, et al. (2011) Developments on drug discovery and on new therapeutics: Highly diluted tinctures act as biological response modifiers. BMC complementary and alternative medicine 11: 101.

69. Chattopadhyay P, Huff R, Shekunov BY (2006) Drug encapsulation using supercritical fluid extraction of emulsions. Journal of Pharmaceutical Sciences 95: 667-679.

70. Yang L, Zhao J, Milutinovic PS, Brosnan RJ, Eger El, et al. (2007) Anesthetic properties of the ketone bodies $\beta$-hydroxybutyric acid and acetone. Anesthesia \& Analgesia 105: 673-679.

71. Nnenna FP, Lekiah P, Obemeata O (2011) Degradation of antibiotics by bacteria and fungi from the aquatic environment. J Toxicol Environ Health Sci 3: $275-285$.

72. Augsburger LL (2003) Tablets and capsules: Design and formulation Dissolution of Dosage Forms. PHAR 535: Pharmaceutics spring.

73. Horsberg TE (1994) Experimental methods for pharmacokinetic studies in salmonids. Annual Review of Fish Diseases 4: 345-358.

74. Verma P, Thakur AS, Deshmukh K, Jha AK, Verma S (2010) Routes of drug administration. International Journal of Pharmaceutical Studies and Research 1: 54-59.

75. Samuelsen OB, Lunestad BT (1996) Bath treatment, an alternative method for the administration of the quinolones flumequine and oxolinic acid to halibut Hippoglossus hippoglossus, and in vitro antibacterial activity of the drugs against some Vibrio sp. Diseases of Aquatic Organisms 27: 13-18.

76. Doggrell S (2012) Introduction to pharmacology, and routes of drug administration. Pharmacology in one semester. Sheila Doggrell.

77. Schellack G (2011) Series on nursing pharmacology and medicine management Part 3: drug dosage forms and the routes of drug administration: nursing pharmacology and medicine management. Professional Nursing Today 15: 10-15.
78. Turner PV, Brabb T, Pekow C Vasbinder MA (2011) Administration of substances to laboratory animals: routes of administration and factors to consider. Journal of the American Association for Laboratory Animal Science: JAALAS 50: 600 .

79. Rosas-Ledesma P, Leon-Rubio JM, Alarcon FJ, Morinigo MA, Balebona MC (2012) Calcium alginate capsules for oral administration of fish probiotic bacteria: Assessment of optimal conditions for encapsulation. Aquaculture Research 43: 106-116.

80. Kosoff RE, Chen CY, Wooster GA, Getchell RG, Bowser PR, et al. (2009) Florfenicol residues in three species of fish after 10-day oral dosing in feed. Journal of Aquatic Animal Health 21: 8-13.

81. Kulkarni P, Chaudhari GH, Sripuram V, Banote RK, Kirla KT, et al. (2014) Oral dosing in adult zebrafish: proof-of-concept using pharmacokinetics and pharmacological evaluation of carbamazepine. Pharmacological Reports 66 : 179-183.

82. Vik-Mo FT, Bergh O, Samuelsen OB (2005) Efficacy of orally administered flumequine in the treatment of vibriosis caused by Listonella anguillarum in Atlantic cod Gadus morhua. Diseases of aquatic organisms 67: 87-92.

83. Kinkel MD, Eames SC, Philipson LH, Prince VE (2010) Intraperitoneal injection into adult zebrafish. Journal of visualized experiments: JoVE.

84. Rottmann RW, Shireman JV, Chapman FA, Brunson MW (1991) Hormone preparation, dosage calculation, and injection techniques for induced spawning of fish. Southern Regional Aquaculture Center.

85. Herman RL (1972) The principles of therapy in fish diseases. Symposium of the Zoological Society of London 30: 141-151.

86. Yanong RP (2006) Use of antibiotics in ornamental fish aquaculture. Circular 84 of the Department of Fisheries and Aquatic Sciences, Florida Cooperative Extension Service, Institute of Food and Frank-Peterside et al. 285 Agricultural Sciences, University of Florida, USA. 